\title{
Cardiovascular Toxicity Associated with Recreational Use of Diphenylprolinol (diphenyl-2-pyrrolidinemethanol [D2PM])
}

\author{
Satnam Lidder, MDa, Paul I. Dargan, FRCPEa, Michael Sexton, MBb , Jenny Button DipFMSc, \\ John Ramseyd, David W Holt, PhDe, David M. Wood, MDa
}

aGuy's and St Thomas' Poisons Unit, London, UK

bEmergency Department, Guy's and St Thomas' NHS Foundation Trust, London, UK

'Forensic Toxicology Service, Analytical Unit, St George's, University of London, London, UK

dTICTAC Communications Ltd, St George's, University of London, London, UK

eAnalytical Unit, St George's, University of London, London, UK

\section{ABSTRACT}

Introduction: Many countries have specific legislation, such as the Controlled Substances Act (1970) in the United States and the Misuse of Drugs Act (1971) in the United Kingdom to control recreational drugs. There is a growing market and supply of "novel" recreational drugs that are not covered under appropriate legislation, despite having similar chemical structures and/or clinical effects. In addition, these novel drugs are often sold legally on the street or through the Internet, with limited details of the exact contents, making application of the appropriate legislation difficult.

Case Report: A male patient with no risk factors for ischemic heart disease, presented to our emergency department with agitation and chest pain characteristic of ischemia following the ingestion of two units of "Head Candy." He improved with oral diazepam over a period of 12 hours and there was no biochemical evidence of myocardial damage. Serum analysis demonstrated the presence of diphenylprolinol (diphenyl-2-pyrrolidinemethanol [D2PM]) and glaucine at concentrations of $0.17 \mathrm{mg} / \mathrm{L}$ and $0.10 \mathrm{mg} / \mathrm{L}$, respectively. No other recreational drugs were detected in an extensive toxicological screen of blood and urine samples.

Discussion: This is the first reported case of confirmed toxicity associated with recreational use of diphenylprolinol in combination with glaucine. In our view, this case provides further support for the need for a systematic approach to toxicological screening of patients with recreational drug toxicity to identify emerging drugs and provide evidence for legislative authorities to assist in revising the legal status of recreational drugs.

Keywords: diphenylprolinol, diphenyl-2-pyrrolidinemethanol (D2PM), recreational drugs, toxicological screening

Acknowledgements: We thank Greg Coumbarides, School of Biological and Chemical Sciences, Queen Mary, University of London, for confirming the identity of the unusual compounds, D2PM and glaucine, from tablets, prior to this study by NMR spectroscopy.

Notes: This case was been presented (but not published) as part of a larger series of "novel" recreational drugs at the 6th Asia Pacific Association of Medical Toxicology (APAMT) meeting in Bangkok, Thailand.

David Wood and Paul Dargan have acted as scientific advisors to the UK Advisory Council on the Misuse of Drugs (ACMD) and the European Monitoring Centre for Drugs and Drug Abuse (EMCDDA).

There was no outside funding of any kind used for this study.

Corresponding Author: Dr. David Wood, Locum Consultant Physician and Clinical Toxicologist, Guy's and St Thomas' Poisons Unit, Avonley Road, London SE14 5ER, UK. Email: David.Wood@gstt.nhs.uk 


\section{INTRODUCTION}

Recreational use of drugs is widespread, with established recreational drugs such as MDMA (3,4 methylenedioxymethamphetamine, known on the street as "Ecstasy") and amphetamines being controlled under relevant drug legislation such as the Controlled Substances Act (1970) in the United States and the Misuse of Drugs Act (1971) in the United Kingdom. Despite this legislation, there have been reports of toxicity associated with emerging novel recreational drugs or pharmaceutical agents misused as recreational drugs [1-3]. These drugs often are structurally very similar to controlled substances, thus result in similar clinical features. Although these legal recreational drugs are sold legitimately in street head shops, the product packing may not contain details of the exact compounds contained within the products [2]. This makes changes to legislation concerning legal highs more difficult, since legislative authorities are not only unaware of what products are being marketed, but also have limited or no clinical information on the toxicity of the compounds contained within many of them. We report here the first confirmed case of recreational use of diphenylprolinol (diphenyl-2pyrrolidinemethanol [D2PM]).

\section{CASE REPORT}

A 21-year-old male nonsmoker with no significant past medical history arrived following ingestion of 3 tablets of "Head Candy" purchased in a local street shop. After ingestion, he felt mildly euphoric for several hours, but as the effects were wearing off he had two "sniffs" of "poppers" (usually $n$-butyl nitrite, isobutyl nitrite or a mixture of the two). Approximately 1 hour later he became dizzy and developed right-sided chest pain and associated right-arm heaviness, consistent with ischemic chest pain, which lasted for 1 hour. He went to the emergency department (ED) approximately 2 hours after the chest discomfort resolved. He was not on any regular medications, there was no family history of note, and although he was a regular user of "herbal highs", there was no previous use of cocaine.

On arrival in the ED, he was agitated with a heart rate of 126 bpm and blood pressure of 213/109 $\mathrm{mmHg}$. He was afebrile and had oxygen saturations of $100 \%$ on room air, and no documented evidence of diaphoresis present on admission. Both of his pupils were equally dilated, but reactive to light; the remainder of his neurological examination, including tone, was entirely normal. His admission electrocardiogram (ECG) showed a sinus tachycardia of 112 beats per minute, with normal QRS (90 msec), QT (304 msec) and QTc (415 msec) durations. Initial laboratory biochemicals were normal (potassium $3.8 \mathrm{mmol} / \mathrm{L}$, creatinine 81 $\mu \mathrm{mol} / \mathrm{L}$, magnesium $0.83 \mathrm{mmol} / \mathrm{L}$, glucose $6.6 \mathrm{mmol} / \mathrm{L}$ ) and an arterial blood gas showed no evidence of significant metabolic derangement ( $\mathrm{pH} 7.47, \mathrm{PaCO}_{2} 4.46 \mathrm{kPa}, \mathrm{PaO}_{2} 11.83 \mathrm{kPa}$, bicarbonate $23.9 \mathrm{mmol} / \mathrm{L}$, lactate $2.0 \mathrm{mmol} / \mathrm{L}$ ).

Further advice on his management was sought from clinicians at Guy's and St Thomas' Poisons Unit, who suggested initial treatment with benzodiazepines for both his agitation and hypertension. Additionally, in view of his symptoms and recent use of volatile nitrites, they recommended that his methemoglobin concentration be measured; this was normal $(0.5 \%)$. He was admitted for overnight observation and treated with $20 \mathrm{mg}$ of oral diazepam, given in divided doses of $2-4 \mathrm{mg}$ over the first 12 hours. His symptoms, agitation, and hypertension resolved with this management. On review by the Clinical Toxicology team the following morning, he was asymptomatic. In view of his chest discomfort he had a repeat 12-lead ECG and a 12-hour Troponin $\mathrm{T}$ concentration, both of which were normal. He was discharged home with advice on recreational drugs and their use.

\section{Toxicological screening}

Informed consent was obtained from the patient for toxicological analysis of blood and urine samples collected on admission; these were sent to the Analytical Unit at St George's, University of London, UK. Routine toxicological analysis of the blood and urine specimens, using full-scan electron ionization gas chromatography-mass spectrometry (GC/MS) after liquid/liquid extraction, identified the presence of D2PM and glaucine, with blood concentrations estimated at $0.17 \mathrm{mg} / \mathrm{L}$ and $0.10 \mathrm{mg} / \mathrm{L}$, respectively. No other drugs or alcohol were detected using a broad toxicology screen of both the blood and urine samples.

\section{DISCUSSION}

We have described the case of an individual who developed ischemic-sounding chest pain and sympathomimetic toxicity following ingestion of legally purchased Head Candy. Subsequent toxicological analysis demonstrated that he had, in fact, ingested both D2PM and glaucine. Glaucine has been reported to cause dissociative-type symptoms both with therapeutic and recreational use $[2,4,5]$. Previous reports of glaucine toxicity do not describe sympathomimetic features, and from its chemical structure and mechanisms of action one would not expect sympathomimetic features with glaucine use $[2,4-6]$. We are not able to interpret the concentrations of D2PM and glaucine detected in this patient, since there is little or no published data with which to compare them.

Methods for the syntheses of D2PM are freely available on the Internet [7]. D2PM is structurally similar to pipradrol, a drug initially developed to treat obesity, but due to its CNS stimulant properties and abuse potential it has largely been removed from the market and controlled under drug legislation since the 1970s $[8,9]$. A patent application for D2PM reported that both the $\mathrm{R}(+)$ and $\mathrm{S}(-)$ isomers of D2PM have been shown to have activity at the cocaine binding site on the dopamine transporter protein [10]. This patent application suggests that these molecules may be useful in treating cocaine addiction, since although they bind to this cocaine binding site with comparable $\mathrm{Ki}_{\text {binding }}$ to cocaine, the $\mathrm{Ki}_{\text {uptake }}$ is significantly higher, suggesting that they have little biological activity. There is no published literature on animal or human studies looking at this potential role of these molecules. 
There is a single-user "trial" of D2PM published on a recreational drug website [11]. In this report the individual ingested $25 \mathrm{mg}$ of what was believed to be (R)(+)-diphenyl-2-pyrrolidinylmethanol, followed by $20 \mathrm{mg} 6$ hours later. Within 2 hours of ingestion the user describes euphoria similar to that seen with amphetamines, with associated jaw clenching, babbling speech and dilated pupils; this individual denied the presence of any heart "rushes" during D2PM use. There has been a recent media report in the nonmedical press of an individual who, following ingestion of "Neuroblast" purchased from "London Underground" (an Internet-based website selling legally available recreational drugs), developed "a surge of adrenalin into my heart," hypertension, and difficulty breathing [12]. The newspaper article noted that subsequent toxicological analysis of the pills showed that they contained D2PM (referred to as diphenyl prolinol) [12].

Extensive toxicological screening did not demonstrate the presence of any other drugs (recreational or prescribed) that could have contributed to our patient's symptoms. In particular, there was no evidence of cocaine use that could have lead to coronary artery vasospasm, myocardial ischemia and the ischemic-sounding chest pain, and no history of previous regular cocaine use. Although the patient described here also reported inhaling a small amount of a volatile nitrite prior to developing his symptoms, we do not feel that this was a significant cause of his symptoms as there was no evidence of methemoglobinaemia on presentation to the ED and at this time his symptoms were still present.

We have already reported that proactive ad hoc toxicological screening undertaken in patients who present following the use of legal highs or in those with atypical presentations following ingestion of classical recreational drugs such as MDMA or amphetamines can detect novel recreational drugs such as glaucine, 2,5-dimethoxy-4-chloroamphetamine (DOC) and 1benzylpiperazine [1-3]. Currently, in the majority of the cases of recreational drug toxicity, routine toxicological screening is not undertaken since real-time results that will alter the individual patient's clinical management are not routinely available. Additionally, in some countries the cost of this screening would be borne by individual patients, rather than through centralized health-service funding. However, this case provides further support for the argument that a more systematically funded approach to toxicological screening in patients with recreational drug toxicity is required, particularly in areas in which the prevalence of recreational drug use is high [13]. This would not only identify novel or emerging drugs, but also provide a more systematic means of monitoring trends of recreational drug use. It would also provide evidence for legislative authorities to assist in revising the legal status of individual compound(s) and the framework under which recreational drugs are controlled.

The authors have no potential financial conflicts of interest to report.

\section{REFERENCES}

1. Wood DM, Dargan PI, Button J, Holt DW, Ovaska H, Ramsey J, et al. Collapse, reported seizure-and an unexpected pill. Lancet 2007;369:1490.

2. Dargan PI, Button J, Hawkins L, Archer J, Ovaska H, Lidder S, et al. Detection of the pharmaceutical agent 'Glaucine' as a recreational drug. Eur J Clin Pharmacol 2008;64:553-554.

3. Ovaska H, Wood DM, Viljoen A, Button J, Puchnarewicz M, Greene SL, et al. 4-chloro-2,5-dimethoxy amphetamine (DOC): A rarely reported rave scene drug of abuse. Clin Toxicol 2007;45:647.

4. Rovinskia VI. A case of hallucinogen-like action of glaucine. Klin Med (Mosk) 1989;67:107-108.

5. Rovinskia VI. Acute glaucine syndrome in the physician's practice: the clinical picture and potential danger. Klin Med (Mosk) 2006;84:68-70.

6. Asencio M, Hurtado-Guzmán C, López JJ, Cassels BK, Protais P, Chagraoui A. Structure-affinity relationships of halogenated predicentrine and glaucine derivatives at D1 and D2 dopaminergic receptors: halogenation and D1 receptor selectivity. Bioorg Med Chem 2005;13:3699-3704.

7. Rhodium site archive hosted on The Vaults of Erowid [webpage on the Internet]. Synthesis of diphenyl-2-pyrrolidinylmethanol and diphenyl-2-pyrrolidinyl-methane [updated 2005 May 31; cited 2008 Feb 19] Available from: http://www.erowid. org/archive/rhodium/chemistry/pyrrolidinyl.html

8. Wikipedia-The Free Encyclopedia [webpage on the Internet]. Pipradrol [updated 2008 Feb 11; cited 2008 Feb 19]. Available from: http://en.wikipedia.org/wiki/Pipradrol

9. Gelvin EP, McGavack TH, Kenigsberg S. Alpha(2-piperidyl) benzhydrol hydrochloride (pipradrol) as an adjunct in the dietary management of obesity. N Y State J Med 1955;55:2336-2338.

10. Pyrrolidine derivatives US patent application [webpage on the Internet]. Patent application by Jackson PF and Guildford Pharmaceuticals; Patent number 5,650,521 [updated 1997 July 22; cited 2008 Feb 19]. Available from: http://v3.espacenet.com/ origdoc?CY=gb\&LG=en\&DB=EPODOC\&IDX=US5650521\& DOC $=$ dcb65d04ab6724e097530cc48c44ebbb62

11. Drugs-Forum research chemicals discussion forum [webpage on the Internet]. Diphenyl-2-Pyrrolidinyl-Methanol (diphenylprolinol) trip reports [updated 2007 Apr 1; cited 2008 Feb 19]. Available from: http://www.drugs-forum.co.uk/forum/ showthread.php? $\mathrm{t}=41534$

12. Gower P [webpage on the Internet]. Herald investigation: Party pill pushers beat the ban [updated 2007 Nov 3; cited 2008 Feb 19]. Available from: http://www.nzherald.co.nz/category/ story.cfm?c_id=30\&objectid=10473827

13. Staack RF. Piperazine designer drugs of abuse. Lancet 2007;369:1411-1413. 